\title{
Cellulose Acetate Blends - Effect of Plasticizers on Properties and Biodegradability
}

\author{
Vu Thanh Phuong ${ }^{1,2}$, Steven Verstichel ${ }^{3}$, Patrizia Cinelli ${ }^{1,4}$, Irene Anguillesi ${ }^{1}$, Maria-Beatrice Coltelli ${ }^{1}$ and \\ Andrea Lazzeri ${ }^{*, 1}$ \\ ${ }^{1}$ University of Pisa, Department of Civil and Industrial Engineering, Via Diotisalvi 2, 56126, Pisa, Italy \\ ${ }^{2}$ Can Tho University, Department of Chemical Engineering, 3/2 Street, Can Tho City, Vietnam \\ ${ }^{3}$ OWS N.V., Dok Noord 5, 9000 Gent, Belgium \\ ${ }^{4}$ National Interuniversity Consortium of Materials Science and Technology, Via G. Giusti 9, 50121, Florence, Italy
}

Received December 20, 2013; Accepted February 19, 2014

\begin{abstract}
Cellulose acetate (CDA) cannot be processed as raw material because it starts to decompose before melting. Triacetin and diacetin were tested to improve CDA processing versus conventional phthalate as environmentally sustainable plasticizers, because of their low toxicity and fast biodegradability. The addition of triacetin and diacetin allowed melt processing of CDA and the results of tensile tests outlined their effect as plasticizers. The values of mechanical properties were compatible with the requirements for applications in rigid packaging. From the results of biodegradation tests it can be concluded that for pure cellulose acetate, complete biodegradation was obtained within 200 days of testing after reinoculation. Incomplete biodegradation was observed for test items with $20 \%$ triacetin or with $30 \%$ phthalate. After 46 days of incubation, the test samples with $30 \%$ plasticizer based on triacetin or triacetin-diacetin were completely biodegraded. These formulations can be selected for the production of compostable blends and/or biocomposites.
\end{abstract}

KEYWORDS: Biodegradation, cellulose acetate, mechanical properties, plasticizers

\section{INTRODUCTION}

The use of plastic materials is widespread in everyday life for diverse applications. Most of the plastics for common use are currently produced from fossil fuels and consumed and discarded into the environment, taking a very long time to degrade and causing the related problems of collection and disposal, often ending up in landfills or in waste incinerators for energy recovery. The increasing pressure on manufacturers by new environmental and waste management policies and consumer demand, in addition to the escalation of oil prices, are steering the trends of polymer technology away from traditional materials towards materials produced by renewable resources. For these reasons, the new field of biodegradable and biobased polymers, which have some environmentally-friendly properties (materials in which the production is based on renewable resources, characterized by low energy consumption, low $\mathrm{CO}_{2}$ emissions, with possibility for composting, biomethanation or recycling), has received growing consideration, which has so far been focused specifically on starch-based products, PLA (Polylactic acid), PHA (Poly hydroxyl alkanoates) in particular PHB (Poly hydroxyl butyrate), cellulose-derived plastics [1], etc. The production of these materials is based on annually renewable agricultural and biomass feedstock. Moreover, biopolymers derived from natural sources can capture markets currently dominated by products based exclusively on petroleum feedstock [2, 3]. However, biodegradable polyesters like PLA and PHA generally present some disadvantages over oilbased plastics such as low fracture toughness, low glass transition temperature $(\mathrm{Tg})$, high moisture absorption, difficult processing, as well as limited applications.

Cellulose acetate (CDA), the most important cellulose-derived biopolymer from an industrial point of view, is a thermoplastic material produced through the esterification of cellulose. A variety of raw materials such as cotton, recycled paper, wood cellulose, and

*Corresponding author: a.lazzeri@ing.unipi.it 
sugarcane are used for producing cellulose esters in powder form $[4,5]$.

Cellulose acetate has been reported to be potentially biodegradable [6]. Therefore, new applications of CDA may be envisaged not only for the packaging, automotive, and electronics industries, but also for medical and pharmaceutical applications, biocompatibility assessment of full blends in the production of biocomposite materials and for other bio-related fields.

Cellulose acetate possesses high glass transition temperature $(\mathrm{Tg})$ and cannot be melt-processed as raw material because it starts to decompose before melting. Therefore, CDA requires the use of plasticizers to reduce its $\mathrm{Tg}$ and processing temperature.

Traditionally plasticization of CDA has been accomplished using citrates, phthalates, glycerol derivatives, phosphates, etc. Phthalate esters, historically the most common industrially used plasticizers for CDA, have been subjected to environmental scrutiny as a health threat, and thus there is now a serious concern about their long-term use. In order to improve the processing of CDA, some studies have explored new plasticizers such as poly(caprolacton triol) [7], polyethylene glycol, propylene glycol [8]. Others considered maleic anhydride, glycol and triacetin (TA) as multi-plasticizers [9]. Their employment research improved the processing of CDAbased materials but at the expense of their mechanical properties. On the basis of the examined literature and Triacetin (TA) and Diacetin (DA) were chosen to improve CDA processing in this study, because they are environmentally sustainable ("eco-friendly") plasticizers with low toxicity and fast biodegradability $[10,11]$

\section{EXPERIMENTAL}

Pellets of plasticized cellulose acetate (CDA, CAS \# 9004-35-7) type Eastman PP-200, with a degree of substitution (DS) of 2.4, were supplied by GIBAPLAST s.a.s. Gazzada Schianno (VA), Italy. Triacetin (glycerin triacetate or 1,2,3-triacetoxypropane, CAS 102-76-1),
Diacetin (Glycerol diacetate or 1,2,3-propanetriol diacetate CAS 25395-31-7), and Diethyl phthalate (CAS 84-66-2) had been provided by Polynt S.p.A., Cavaglià (BI) Italy. The plasticized compounds have been produced by melt mixing in a Bandera single screw extruder with screw diameter of $45 \mathrm{~mm}$. The granules were subsequently used for biodegradation tests without further thermal treatment.

Plasticized CDA granules have been processed in a MiniLab II Haake Rheomex CTW 5 conical twin-screw extruder (Thermo Scientific Haake $\mathrm{GmbH}$, Karlsruhe, Germany), at a screw rate of $80 \mathrm{rpm} / \mathrm{min}$ in a temperature range from $170^{\circ} \mathrm{C}$ to $210^{\circ} \mathrm{C}$, depending on the material formulations (Table 1). After extrusion, the molten materials were immediately transferred through a preheated cylinder to the Haake MiniJet II mini injection molder (Thermo Scientific Haake $\mathrm{GmbH}$, Karlsruhe, Germany), to obtain using the Haake type 3 specimen (557-2290) dog-bone tensile bars. The specimens were placed in plastic bags for vacuum sealing to prevent moisture absorption. The samples produced in this way were used for dynamical mechanical testing. Samples for tensile testing were injection molded using a Negri Bossi (Cologno Monzese, Italy) press into ISO 3167 multipurpose specimens c/o LPM srl (Scandicci, Italy).

Tensile tests were performed at room temperature, at a crosshead speed of $10 \mathrm{~mm} / \mathrm{min}$, by means of an Instron 5500R universal testing machine (Canton, MA, USA) equipped with a $10 \mathrm{kN}$ load cell and interfaced with a computer running the Merlin software (Canton, MA, USA). The Young modulus was determined using an Instron extensometer with gauge length of $12.5 \mathrm{~mm}$. Dynamic mechanical thermal analysis (DMTA) was carried out on a Gabo Eplexor ${ }^{\circledR}$ 100 N (Gabo Qualimeter GmbH, Ahlden, Germany). Test bars were cut from the type 3 tensile bar specimens (size: $20 \times 5 \times 1.5 \mathrm{~mm}$ ) and mounted in tensile geometry. The temperature used in the experiment ranged from $-100^{\circ} \mathrm{C}$ to $170^{\circ} \mathrm{C}$, at a heating rate of $2^{\circ} \mathrm{C} / \mathrm{min}$ and frequency of $1 \mathrm{~Hz}$.

Table 1 Composition of the samples produced.

\begin{tabular}{lcccc}
\hline Sample & $\begin{array}{c}\text { Cellulose acetate } \\
\mathbf{( \% )}\end{array}$ & $\begin{array}{c}\text { Triacetin } \\
\mathbf{( \% )}\end{array}$ & $\begin{array}{c}\text { Diacetin } \\
\mathbf{( \% )}\end{array}$ & $\begin{array}{c}\text { Phthalate } \\
\mathbf{( \% )}\end{array}$ \\
\hline CDA & 100 & - & - & - \\
CDA80TA20 & 80 & 20 & - & - \\
CDA70TA30 & 70 & 30 & - & - \\
CDA70DA30 & 70 & - & 30 & \\
CDA70T15DA15 & 70 & 15 & 15 & - \\
CDA70PH30 & 70 & - & - & 30 \\
\hline
\end{tabular}


The biodegradation of the samples was examined under controlled composting conditions according to the international standard ISO 14855-1 (2012), Determination of the ultimate aerobic biodegradability of plastic materials under controlled composting conditions - Method by analysis of evolved carbon dioxide $[12,13]$. The controlled composting biodegradation test is an optimized simulation of an intensive aerobic composting process where the biodegradability of a test item under dry aerobic conditions is determined. The inoculum consists of stabilized and mature compost ( $>20$ weeks) derived from the organic fraction of municipal solid waste (MSW). Before use, the compost was sieved over $5 \mathrm{~mm}$, with the fine fraction used as inoculum. The biodegradation test was performed in duplicate and control, reference and test item reactors were included. Microcrystalline cellulose powder (Avicel; Merck, Darmstadt, Germany) was used as the positive reference. The CDA samples were cryogenically milled till dimensions $<800 \mu \mathrm{m}$. The control reactors contained $1200 \mathrm{~g}$ of compost inoculum. Reference and test samples were mixed with the inoculum in a defined ratio ( $80 \mathrm{~g}$ sample/1200 compost) and introduced into the reactors. The reactors were placed in an incubator at $58 \pm 2^{\circ} \mathrm{C}$. Air was passed over a gas flow controller, which regulates very precisely the flow rate, and blown into the composting vessel at the bottom through a porous plate. The gas leaving each individual reactor was continuously analyzed at regular intervals for $\mathrm{CO}_{2}$ and $\mathrm{O}_{2}$. At the same moment the flow rate was measured. The percentage of biodegradation was determined as the percentage of solid carbon of the test compound that was converted to gaseous, mineral $\mathrm{C}$ in the form of $\mathrm{CO}_{2}$. During the incubation, the whole content of the reactors was shaken manually on a weekly basis, so that air channels and fungal flocks were broken up and moisture, microbiota and substrate were divided evenly. At the same time, the moisture content was checked and adjusted if necessary by dry or wet aeration or by adding water directly to the reactors. The total test duration was 200 days. After 46 days the test was stopped for all CDA samples with plasticizer. At the same time the test setup was re-inoculated with 10\% fresh VGF (Vegetable, Garden and Fruit) waste. By this interaction, extra nutrients and new microbial flora were supplied in an attempt to increase the biodegradation rate of CDA. After 136 days, a new and strong reinoculation with $20 \%$ fresh VGF was executed.

\section{RESULTS AND DISCUSSION}

A few researchers used a melt processing approach to improve the performance of cellulosic plastics [14]. In the present paper, CDA was extruded with triacetin (TA) and diacetin (DA) and the content of plasticizers used was varied from $20 \%$ to $30 \%$ by weight (wt $\%$ ) as reported in Table 1.

In fact, based on our preliminary testing [15], it is not possible to process CDA with less than $20 \mathrm{wt} \%$ of plasticizers, since for lower plasticizer contents the melt processing temperature of the plasticized CDA exceeds its decomposition temperature. Preliminary experiments in our lab also showed that exceeding $30 \mathrm{wt} \%$ plasticizers, for example, with $40 \mathrm{wt} \%$ of TA, the main mechanical properties (elastic modulus, tensile strength, and elongation at break) of materials become unacceptably low. This can be explained by the fact that the functional groups of CDA are completely solvated by triacetin when the TA or DA content is over $30 \%$. Further increases in plasticizer concentrations just separate the individual CDA molecules, decreasing their interchain interactions. A thermodynamic study has shown that the enthalpy for mixing is exothermal over the whole range of compositions and the Gibbs energy of the mixing of the CDA/TA blend has a minimum corresponding to about $40 \mathrm{wt} \%$ of TA [16]. Also a fall in entropy of the system was found following the formation of solvates between the functional groups of CDA and triacetin for TA concentrations up to about $40 \mathrm{wt} \%$, followed by an increase in the entropy of the system as a result of disturbance of the interchain interaction in CDA on its solvation for larger concentrations.

Mechanical properties of the preparation blends, after one day and 180 days (six months) respectively, are reported in Table 2.

As reported before, it was not possible to process CDA without plasticizers; with 20 wt $\%$ of TA it was possible to produce samples by melt processing with relatively limited elongation at break and high strength and modulus. In order to produce samples with higher flexibility still suitable for some applications in rigid and semi-rigid plastic, blends were prepared with a total content of $30 \mathrm{wt} \%$ of plasticizers. The solubility of TA and DA in CDA is high since TA and DA are relatively low viscosity liquids that can easily diffuse into CDA, thus they both result in efficient plasticizers of CDA, enabling a larger processing window for plasticized CDA, and increasing the flexibility of the material. At the same content (30 wt\%) TA and DA were almost as efficient as phthalate as a plasticizer. There was no evident advantage from the use of mixtures of TA and DA, since the sample with $15 \mathrm{wt} \%$ of TA and DA, respectively, presented slightly lower values in strength and modulus. With aging of the samples for six months at room temperature and $50 \% \mathrm{RH}$, no leaching of plasticizers was observed and the mechanical properties varied in the limits of the standard deviation for this type of measurements, thus the blends appear stable in properties. 
Vu Thanh Phuong et al.: Biodegradable Cellulose Acetate Blends

Table 2 Mechanical properties of the prepared Blends.

\begin{tabular}{lccc}
\hline Sample & Elongation at Break (\%) & Tensile Strength $(\mathbf{M P a})$ & Young's Modulus (GPa) \\
\hline CDA80TA20 & $8.3 \pm 3.1$ & $58.3 \pm 4.7$ & $3.1 \pm 0.1$ \\
CDA70TA30 & $11.6 \pm 1.8$ & $30.2 \pm 0.2$ & $2.1 \pm 0.1$ \\
CDA70DA30 & $9.4 \pm 2.4$ & $27.2 \pm 0.2$ & $1.9 \pm 0.1$ \\
CDA70TA15DA15 & $11.0 \pm 0.1$ & $24.7 \pm 0.2$ & $1.7 \pm 0.1$ \\
CDA70PH30 & $16.8 \pm 0.4$ & $30.4 \pm 0.4$ & $2.0 \pm 0.1$ \\
After 6 Months & & & \\
CDA80TA20 & $9.9 \pm 4$ & $57.2 \pm 0.8$ & $3.3 \pm 0.1$ \\
CDA70TA30 & $13.3 \pm 2.3$ & $31.5 \pm 1.5$ & $2.2 \pm 0.1$ \\
CDA70DA30 & $7.3 \pm 2.7$ & $26.1 \pm 2.1$ & $1.9 \pm 0.2$ \\
CDA70TA15DA15 & $11.9 \pm 0.7$ & $25.8 \pm 0.2$ & $1.9 \pm 0$ \\
CDA70PH30 & $24.4 \pm 1.0$ & $32.0 \pm 0.25$ & $2.2 \pm 0.1$ \\
\hline
\end{tabular}

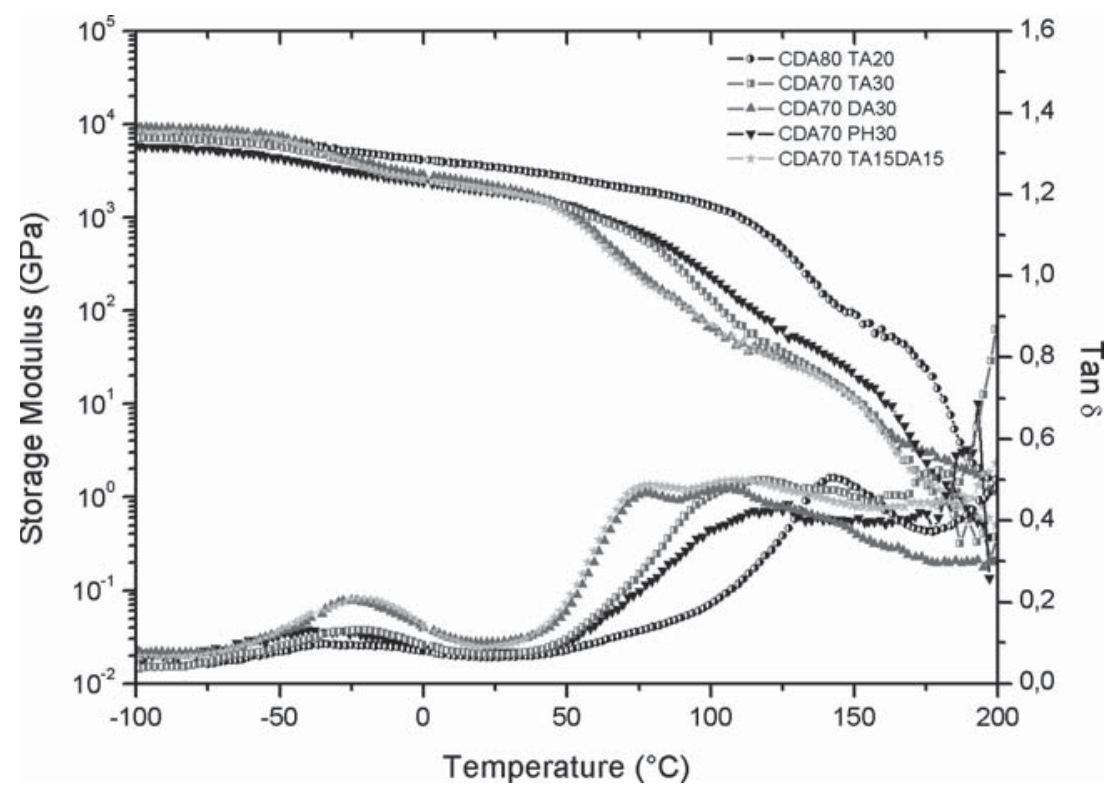

Figure 1 Dynamic Mechanical Thermal Analysis of CDA based blends.

Figure 1 presents the DMTA thermogram for cellulose acetate and its blends with different type and content of plasticizers. The temperature dependence of storage modulus and loss tangent is shown in this figure. Two major transitions can be observed for all the blends at 20 and $30 \%$ by weight of plasticizer. The high temperature peak corresponds to the glass transition of CDA $[17,18]$. The addition of plasticizer leads to a linear decrease in the $\alpha$-transition temperature of CDA.

The low temperature peak at $-28^{\circ} \mathrm{C}$, normally defined as the $\beta$-peak, is sometimes associated with the movement of the glucose ring units or to water associated with hydroxymethyl groups [17]. Thus the molecules of the plasticizer intercalate between the chains of polymers, spacing them apart and increasing the "free volume."
This increased space between polymer molecules enables the reduction of the activation energy for the cooperative motions of the main chain and has the effect of significantly lowering the glass transition temperature of CDA. This effect is more significant with a content of plasticizer of $30 \%$ by weight and, in particular, for the samples with $30 \%$ of DA (CDA70DA30) and with $15 \%$ of both DA and TA (CDA70DA15TA15), which present the lowest value for the CDA $\alpha$ transition; and the $\beta$ peak at $-28^{\circ} \mathrm{C}$ is much more pronounced than in the other samples.

The mechanical and thermal properties of these blends are compatible with application in rigid and semi-rigid packaging, but an important factor for application of biodegradable packaging is related 
to the time requested for biodegradability in compost. Thus it was important to evaluate the biodegradation of the samples under controlled composting conditions. In this test the biodegradation of the reference material cellulose started almost immediately and reached a biodegradation of $92.1 \%$ after 46 days (Figure 2), which means that the $70 \%$ pass level for a valid test as stated by ISO 14855 was easily obtained and demonstrated the good quality of the inoculum.

The degradation of CDA is influenced by the degree of substitution (DS) [19] and it is reported that the biodegradation rates are reduced, but not inhibited, by a higher level of acetylation; thus Gu [19] demonstrated that CDA films with DS between 1.7 and 2.5 completely disappeared after 7 and 18 days of incubation, respectively, while in a degradation test simulating natural composting conditions including a thermophilic phase, Gardner et al. [20] observed that the weight loss is dependent upon DS with a significantly delayed degradation at DS greater than 2.1. No biodegradation was observed in this study for the pure CDA with DS of 2.4 during the first 46 days of testing. Only after subsequent reinoculation with fresh biowaste $(10 \%$ after 46 days and $20 \%$ after 136 days) biodegradation took off and finally resulted in complete biodegradation after 200 days (Figure 3).

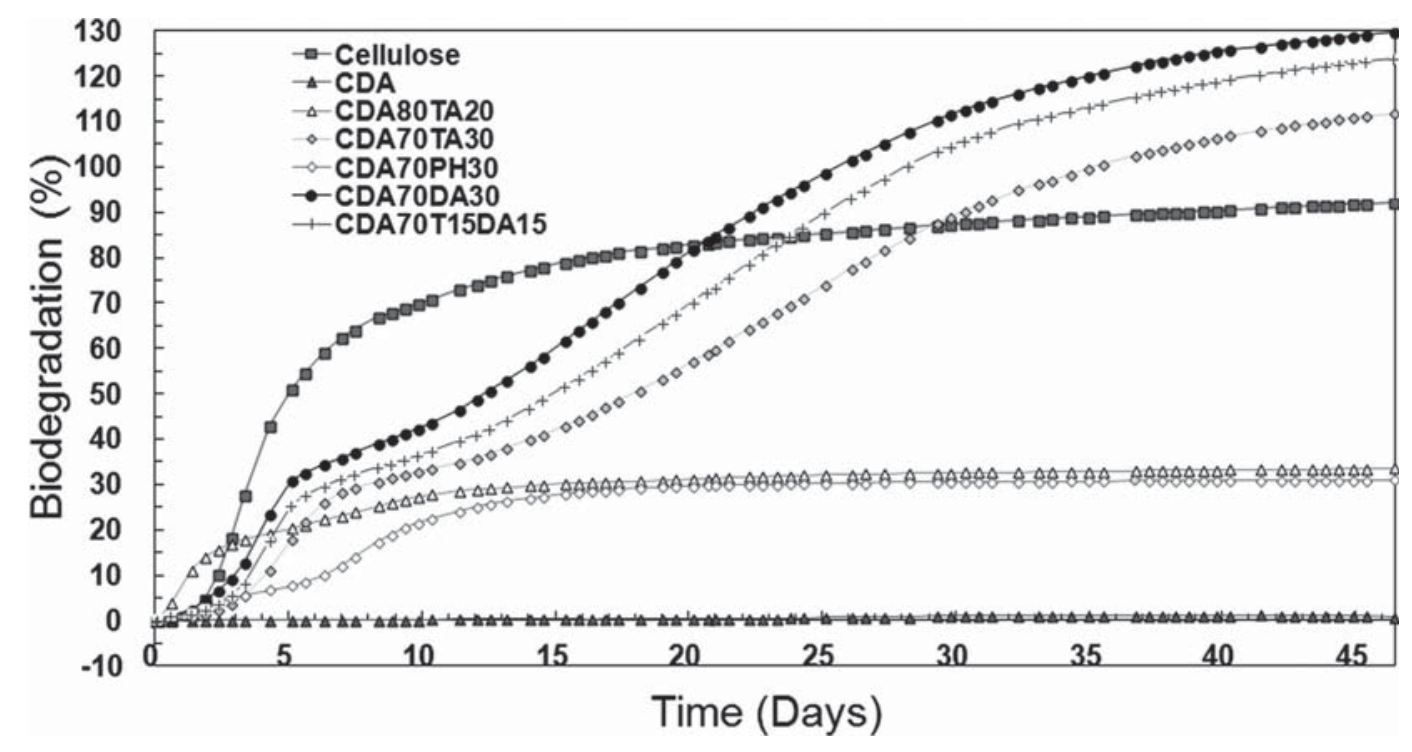

Figure 2 Evolution of biodegradation of samples based on cellulose acetate until 46 days.

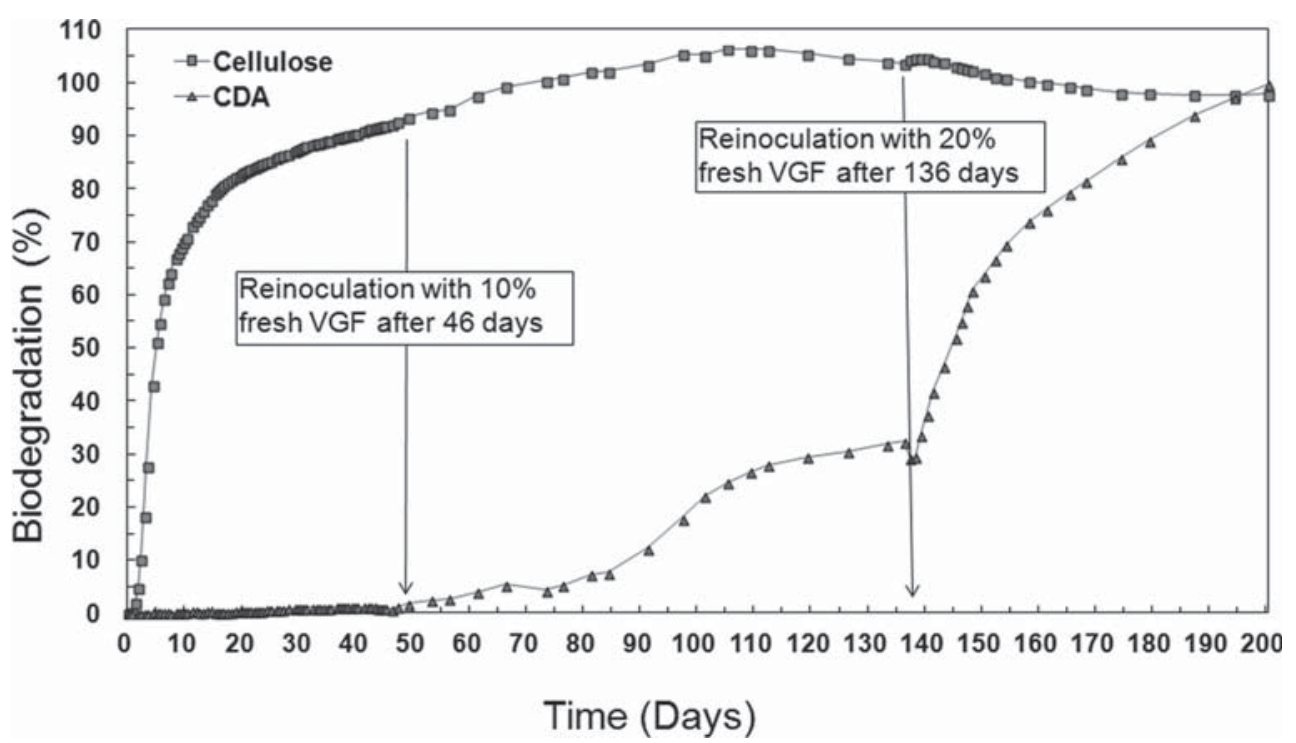

Figure 3 Evolution of biodegradation of samples based on cellulose acetate until 200 days. 
The first step in the degradation of CDA is the deacetylation. Deacetylation might be established partly by heat and high $\mathrm{pH}$ [21]. Addition of fresh biowaste not only introduced additional nutrients and microorganisms, but also changed the $\mathrm{pH}$ of the reactor content and triggered the biodegradation.

In spite of the slow degradation of pure CDA, the CDA samples with plasticizers almost immediately started to degrade. However, after 15 days a plateau in biodegradation was formed for test items Cellulose acetate $80 \%$ / Triacetin $20 \%$ and Cellulose acetate $70 \%$ / Phthalate $30 \%$, while the biodegradation of Cellulose acetate 70\%/Triacetin 30\%, Cellulose acetate 70\%/ Diacetin 30\%, and Cellulose acetate 70\% / Tri+Diacetin $30 \%$ proceeded further at a good rate.

After 46 days a plateau in biodegradation at about $33.6 \pm 2.6 \%$ and $31.1 \pm 3.3 \%$ was measured for test items CDA80TA20 and CDA70PH30, respectively. On a relative basis, compared to the suitable reference substrate cellulose, a biodegradation of $36.4 \%$ and $33.7 \%$ was calculated. As no biodegradation was established for the pure cellulose acetate it can be concluded that the plasticizer was degraded.

Complete biodegradation under controlled composting conditions was observed for test items CDA70TA30, CDA70DA30, and CDA70TA15DA15 at a level of $111.8 \% \pm 1.0 \%, 129.7 \% \pm 0.9 \%$ and $124.0 \% \pm 3.5 \%$, respectively, after 46 days of testing. Values exceeding $100 \%$ are due to a synergistic effect called priming that occurs if the compost inoculum in the test reactor is producing more $\mathrm{CO}_{2}$ than the compost inoculum in the control reactors, resulting in a net $\mathrm{CO}_{2}$ production that is not exclusively coming from the test item and, in case of readily degradable products, in a biodegradation percentage of more than $100 \%$ [22-25]. The addition of plasticizer increased the biodegradation rate of CDA. This was in line with the work of Buchanan [26] that demonstrated that the addition of plasticizer PEG400 has a positive effect on biodegradation of CDA blends. Moreover, in previous studies [15] we observed a decreasing in thermal stability associated with higher content of plasticizer (triacetin) in blends with CDA. Also, the data of the DMTA analysis reported before (Figure 1) outlines a higher plasticizing effect in the blends with $30 \%$ of DA and $30 \%$ of DA/TA $50 / 50$ solution.

\section{CONCLUSIONS}

Both triacetin and diacetin resulted in a potential substitute for phthalate as plasticizers for CDA. With a $30 \%$ content of either triacetin, diacetin or a mixture of both, it is possible to easily process and injection mold specimen based on CDA with mechanical properties compatible with common requirements for rigid applications and interesting for several practical applications such as components for automobiles, electronics, etc., considering the high modulus and thermal stability of CDA versus other biodegradable polymers.

From the results of biodegradation tests it can be concluded that for the pure cellulose acetate, complete biodegradation was obtained within 200 days of testing after reinoculation. The European Norm EN13432 on compostability stipulates maximum test duration of 180 days. Incomplete biodegradation was observed for test items with $20 \%$ triacetin or with $30 \%$ of Phthalate.

The test samples with $30 \%$ plasticizer based on triacetin or triacetin-diacetin were completely biodegraded within 46 days of composting; thus these formulations can be selected for the production of compostable blends and/or biocomposites.

\section{ACKNOWLEDGMENTS}

The support from FP7 - KBBE project no. 212239 Forbioplast (Forest Resource Sustainability through Bio-Based Composite Development) to carry out this research is acknowledged.

\section{REFERENCES}

1. L. Yu, K. Dean, and L. Li, Polymer blends and composites from renewable resources. Prog. Polym. Sci. 31, 576-602 (2006).

2. A.K. Mohanty, M. Misra, and G. Hinrichsen, Biofibers, biodegradable polymers and biocomposites: An overview. Macromol. Mater. Eng. 276, 1-24 (2000).

3. C. Scholz and R.A. Gross (Eds.), Polymers from Renewable Resources-Biopolyesters and Biocatalysis, in ACS Symposium Series, Volume 764, American Chemical Society, Washington, DC. (2001).

4. K.J. Edgar, C.M. Buchanan, J.S. Debenham, P.A. Rundquist, B.D. Seiler, M.C. Shelton, and D. Tindall, Advances in cellulose ester performance and application. Prog. Polym. Sci. 26, 1605-1688 (2001).

5. G. Rodrigues Filho, D.S. Monteiro, C.S. Meireles, R.M.N. Assunção, D.A. Cerqueira, H.S. Barud, S.J.L. Ribeiro, and Y. Messadeq, Synthesis and characterization of cellulose acetate produced from recycled newspaper. Carbohydr. Polym. 73, 74-82 (2008).

6. C.M. Buchanan, R.M. Gardner, and R.J. Komarek, Aerobic biodegradation of cellulose acetate. J. Appl. Polym. Sci. 47, 1709-1719 (1993).

7. M.M. Meier, L.A. Kanis, J.C. de Lima, A.T.N. Pires, and V. Soldi, Poly (caprolactone triol) as plasticizer agent for cellulose acetate films: Influence of the preparation procedure and plasticizer content on the physico-chemical properties. Polym. Adv. Tech. 15, 593-600 (2004). 
8. P.R. Rao and P.V. Diwan, Permeability studies of cellulose acetate free films for transdermal use: Influence of plasticizers. Pharm. Acta. Helv. 72, 47-51 (1997).

9. S.H. Lee and N. Shiraishi, Plasticization of cellulose diacetate by reaction with maleic anhydride, glycerol, and citrate esters during melt processing. J. Appl. Polym. Sci. 81, 243-250 (2001).

10. S.H. Lee, S.Y. Lee, J.D. Nam, and Y. Lee, Preparation of cellulose diacetate/ramie fiber biocomposites by melt processing. Polym. Korea 30, 70-74 (2006).

11. S.Y. Lee, M.S. Cho, J.D. Nam, and Y. Lee, Melting processing of biodegradable cellulose diacetate/starch composites. Macromol. Symp. 242, 126-130 (2006).

12. Determination of the ultimate aerobic biodegradability of plastic materials under controlled composting conditions-Method by analysis of evolved carbon dioxide-Part 1: General method, Standard specification ISO 14855-1 (2012)

13. Standard specification for Packaging-Requirements for packaging recoverable through composting and biodegradation-Test scheme and evaluation criteria for the final acceptance of packaging, EN 13432 (2000).

14. A.K. Mohanty, A. Wibowo, M. Misra, and L.T. Drzal, Development of renewable resource-based cellulose acetate bioplastic: Effect of process engineering on the performance of cellulosic plastics. Polym. Eng. Sci. 43, 1151-1161 (2003).

15. V.T. Phuong and A. Lazzeri, "Green" biocomposites based on cellulose diacetate and regenerated cellulose microfibers: Effect of plasticizer content on morphology and mechanical properties. Compos. A 43, 2256-2268 (2012).

16. I.B. Rabinovich, V.I. Petkov, and S.S. Zarudayeva, Physicochemical analysis of mixtures of cellulose diacetate with triacetin and thermodynamic characteristics of the process of mixing them. Polym. Sci. USSR 27, 2040-2047 (1985).
17. G. Számel, S. Klébert, I. Sajó, and B. Pukánszky, Thermal analysis of cellulose acetate modified with caprolactone. J. Therm. Anal. Cal. 91, 715-722 (2008).

18. M. Scandola and G. Ceccorulli, Viscoelastic properties of cellulose derivatives: 1. Cellulose acetate. Polymer 26, 1953-1957 (1985).

19. J.D. Gu, D.T. Eberiel, S.P. McCarthy, and R.A. Gross, Cellulose acetate biodegradability upon exposure to simulated aerobic composting and anaerobic bioreactor environments. J. Environ. Polym. Degr. 1, 143-153 (1993).

20. R.M. Gardner, C.M. Buchanan, R. Komarek, D. Dorschel, C. Boggs, and A.W. White, Compostability of cellulose acetate films. J. Appl. Polym. Sci. 52, 1477-1488 (1994).

21. J. Puls, S.A. Wilson, and D. Hölter, Degradation of cellulose acetate-based materials: A review. J. Polym. Environ. 19, 152-165 (2010), doi:10.1007/s10924-010-0258-0.

22. J. Shen and R. Bartha, Priming effect of substrate addition in soil-based biodegradation tests, Appl. Environ. Microbiol. 62, 1428-1430 (1996).

23. S. Fontaine, A. Mariotti, and L. Abbadie, The priming effect of organic matter: A question of microbial competition? Soil. Biol. Biochem. 35, 837-843 (2003).

24. F. Degli-Innocenti, M. Tosin, and C. Bastioli, Evaluation of the biodegradation of starch and cellulose under controlled composting conditions. J. Envrion. Polym. Degr. 6, 197-202 (1998).

25. S. Verstichel, B. De Wilde, E. Fenyvesi, and J. Szetjli, Investigation of the aerobic biodegradability of several types of cyclodextrins in a laboratory-controlled composting test. J. Polym. Environ. 12, 47-55 (2004).

26. C.M. Buchanan, D. Dorschel, R.M. Gardner, R.J. Komarek, A.J. Matosky, A.W. While, and M.D. Wood, The influence of degree of substitution on blend miscibility and biodegradation of cellulose acetate blends. J. Environ. Polym. Degr. 3, 179-195 (1996). 